\title{
Multi-Factorial Management of Fall Risk in the Home Setting With Use of Innovative Technology: A Case Study
}

\author{
Rebecca J Tarbert ${ }^{1}$ \\ ${ }^{1}$ ActiveProtective Technologies, Inc, Fort Washington, PA, United States \\ Correspondence: Rebecca J Tarbert, ActiveProtective Technologies, Inc, Fort Washington, PA, United States.
}

Received: September 24, 2020

Accepted: November 10, 2020

Online Published: December 2, 2020

doi:10.20849/ijsn.v5i4.830

URL: https://doi.org/10.20849/ijsn.v5i4.830

\begin{abstract}
Fall risk and fall injury risk are a raising concern for older adults living in the home and community environment Injuries from falls account for reduced independence for older adults in the form of limitations of activities of daily living performance and significant dependency for ambulation. Up to $50 \%$ of those who suffer a hip fracture from a fall will not be able to return to their prior level of living. Serious fall injuries create a necessity to move into a setting where increased levels of care are provided. The ability to remain in the most desired setting of their own home is a critical part of successfully aging in place. According to the Centers of Disease Control, 36 million older adults in the United States suffered a fall in 2018 with 8 million reported injuries. With the expansion of the older adult population, these numbers are anticipated to inflate up to 52 million falls with 12 million reported fall injuries by the year 2030. Several countries have adopted fall risk mitigation programs for fall risk identification in primary care and community provision of fall management strategies. Traditional methods of fall risk management follow the American Geriatrics Society's and British Geriatrics Society's Clinical Practice Guideline. Though great strides are taken to intervene on the modifiable risk factors for falls, the risk of falls for many individuals remains. Introduction of new and innovative technologies can offer increased safety and ability to participate in functional mobility for those most at risk of falls and fall injuries.
\end{abstract}

Keywords: fall risk, fall injuries, hip fracture, hip protection, mobility

\section{Introduction}

As the older adult population grows, there are substantial implications of fall and falls related injuries that impact the social and financial health of our society. According to the CDC, 1 out of 4 adults over the age of 65 will fall each year and many fall repeatedly. The devastating results of falls include physical injuries such as bruises, cuts, broken bones and head injuries bringing over 3 million older adults to the emergency room annually. Experiencing a fall leads not only to need for medical care, but also lead to intrinsic fear of falling and reduced participation in activity for older adults in any setting. For older adults living at home in the community, the frequency of falls increases with age and frailty level. Falls at home occur mostly outside in the yard, but those inside the home occur most often in the bedroom, kitchen and dining room. Identification of fall risk for older adults living in the community has been highlighted as one area of medical assessment in recent years. The Medicare annual wellness visit in the United States includes the recommended questions of "Have you experienced 2 or more falls within the past year or sought medical attention following a fall or feel unsteady when walking?" The NHS in the United Kingdom utilizes the Falls Risk Assessment Tool (FRAT) to screen for fall risk with the general practitioner interaction with patients over the age of 65 . In the home and community setting, normal mitigation strategies for identified fall risk factors include environmental hazard assessment, medication review, vision check and prescription for rehabilitation services to offer remediation and/or compensation. Even with the individualized risk factor identification and management, there is often ongoing risk for falls and fall injuries that remain inherent to the individual due to unmodifiable factors.

\section{Theoretical Background}

Technological advancements in recent years have offered an increased array of devices and methods to promote with those at risk of falls and fall injuries the ability to age in place with increased independence and increased safety. Smart devices that automatically send notifications of physiologic status and changes continuously monitor those who are being managed for chronic conditions. Personal Emergency Response Systems (PERS) have evolved from telephonic pendant press to fall monitoring and GPS elevated tracking to offer clients, caregivers and families increased peace of mind for those at risk individuals living at home alone. Virtual 
platforms are increasing the communication and connection between patients and providers to offer increased safety from infectious disease as well as decreased wait times and increased one on one time patients have with providers due to the elimination of need to leave their own home. Predominately, fall management technologies alert to the need of assistance due to a fall after the fall and in many cases, fall injury has already occurred. For those who are living with a high risk of fragility fracture, a solution to avoid the seriously debilitating and often deadly experience of a hip fracture is needed. Each year, approximately 300,000 adults 65 or older are hospitalized in the United States with hip fracture and this number is expected to grow with the expanding senior population. Outcomes following a hip fracture include significant increase in mortality, greatly impact the person's physical mobility, psychological health and social functioning leading to reduced quality of life. Annual medical costs due to hip fracture are estimated to be greater than $\$ 50,000$ per patient, leading to greater than $\$ 5$ billion to the US healthcare system per year. Emerging technologies with advancing ability to accurately detect human motion are now able to offer on-demand safety technology as never before.

\section{Method}

The following is a case study of a 91-year-old gentleman who lives in the community within a home environment. The retired engineer lives in a suite of the home where his daughter, son-in-law and granddaughter also reside. As a veteran of the United States Navy, he is part of the Veterans Affairs system and utilizes in home monitoring using remote weight, blood pressure and heart rate monitoring daily. His home equipment included the utilization of a PERS pendant, as well as use of voice activated smart device for making telephone calls and the regular use of a desktop computer with access to the internet. His care management includes in home visits with primary care and nursing as needed to manage chronic conditions of heart failure and severe degenerative arthritis. Occupational and physical therapy rehabilitation services were provided to him for treatment diagnoses of pain, history of falls, risk of falls and difficulty with ambulation. The fall risk factors of visual impairment, multiple medications, difficulty with ambulation, fear of falling, limited mobility due to fear of falling, pain and incontinence places this individual at a high risk of falls. Medical management and continual monitoring of medications was implemented for reducing number of medications as well as managing incontinence. The occupational therapy intervention included environmental modifications to offer compensation for the functional impairments of limited ROM, pain, decreased activity tolerance and poor balance. Modifications included: hospital bed, trapeze adaptation for ease of transfer, handles applied to counters and walls for increased stability points in places standing for prolonged periods of time are necessary (bathroom and kitchen), installation of shower bench, manual wheel chair order, fitting and training, and power wheel chair order, fitting and training. Physical therapy intervention provided evidence-based strength training exercises and balance neuromuscular techniques to improve intrinsic balance as well as prescription of home exercise program for core strength, extremity strength, walking program with 4 wheeled walking (primary mode of assistive device for functional mobility) and balance training with family. This cognitively intact older adult continued to express a fear of falling at the time of discharge of therapy services. The rehabilitation providers had addressed all modifiable factors of falling through compensation with environmental adaptations as well as functional training on performance of ADL's with assistive devices for independent and safe performance. His functional status progressed with the following standardized measures taken: (modified) 30 second chair rise $=16$ repetitions no use of UE's (from elevated seat height of 25 inches), Timed Up and Go $=22$ seconds, and self-selected walking speed of .72 meters/second. Though the 30 second chair rise was modified, it was done so to reflect functional leg strength specifically by elevating the seat height versus modifying with use of upper extremities which reflects functional transfer performance. The self-selected walking speed is just under the threshold of fall risk for older adults recognized at .76 meters/second indicating further need of fall risk intervention to reduce risk of adverse events. Timed Up and Go scoring reflects definite fall risk with cut-off scoring recognized as 13.5 seconds for community dwelling adults, but well underneath the cutoff score for frail elderly (32.6 seconds). It was apparent that the provided therapeutic intervention was effective as each of these scores were meaningfully improved upon therapy discharge, though the risk of falls and adverse event from fall remained inherent.

With the sharing of continued fear of injury from falls, recognized risk of falling and strong desire for this older adult to remain in his home setting, the introduction of a safety device was made. The utilization of a smart wearable technology to increase safety as well as maintain mobility was initiated 1 week following discharge of rehabilitation services. The Tango Belt is a fall-sensing wearable technology in the form of a slim belt, offering constant human motion monitoring that can increase the safety from fall related hip fractures and promote mobility for older adults at risk of fragility fracture. Using 3D sensors, the Tango Belt can monitor where the individual is in space and the acceleration of their motion. Upon recognition of an irrecoverable, serious hip-impacting fall, the belt deploys left and right anatomically fitted airbags before the fall impact occurs to protect the hips. In cases of airbag deployment and other fall scenarios, the wearable sensor can also be enabled to send alerting message to caregivers that assistance is needed by the individual. 
Traditional models of offering safety from fall related injuries in senior care includes the utilization of sensors and call buttons that provide care attention after a fall has occurred and the use of passive hip pads. Passive hip pads that are worn under clothing to protect the hip joints in the case of a fall have limited effectiveness due to limited adherence to wear as they are uncomfortable. A wearable technology that activates airbags when the need arises offers on-demand protection during the fall event to absorb the impact force and reduce or eliminate injury. The belt will then automatically alert caregivers that assistance is needed for the wearer who has suffered the fall. The location of the motion sensor in the belt, being centered on the lower back, provides the ability to monitor the balance of the wearer in a truly objective way of postural sway during the performance of their daily activities. Users and caregivers monitoring the data being gathered by the belt sensor will see how many days and hours the belt is being worn by the wearer, track balance confidence with input of the Short version of the FES-I (Falls Efficacy Scale International) and monitor changes in balance via the postural sway meter. These metrics of reporting will offer the caregivers insight to the fall risk of the wearer and ability to screen for possible need of intervention with changes in postural sway.

\section{Outcome}

The individual was fit for the Tango Belt, the connection to WiFi in the home was successful and he was instructed to wear the safety device at his discretion. The Short version of the Falls Efficacy Scale International (FES-I) was taken using the companion app for the Tango Belt and resulted in a score of 18 indicating high perceived concern of falling in the performance of functional activity. 1 week after introduction to the wearable, the client was re-assessed for adherence. Client reported and the monitoring data from the wearable reported continual wear of the Tango Belt up to 24 hours each day. Removal of the safety device occurred only with charging (every $3^{\text {rd }}$ day for 3 hours) and when going to take a shower. A statement from the client was "I don't even feel it on and I don't feel safe without it on. I wear it all the time." In the weeks following initiation of the wearable safety device, the client reported that he "doesn't like to take it (the belt) off" and that he had no pain or skin irritation from wearing it for prolonged periods of time. The client continued to wear the Tango Belt for 8 weeks performing self-directed home exercise programming provided by the physical therapist upon discharge of services as well as regularly attending to a walking program with his daughter as tolerated 3 times a week. The functional metrics were repeated at 8 weeks post device initiation. The Short FES-I score was 17 indicating an improvement in the level of balance confidence by $6 \%$. The 30 second chair rise was repeated in the modified version of seat height elevation to 25 inches with performance reflective of 18 repetitions with no use of upper extremities indicating increased lower extremity strength. Timed Up and Go testing revealed a score of 21.44 seconds and self-selected walking speed increased to .84 meters/second surpassing the cut-off point for fall risk of community-living individual. All functional testing including the self-perceived balance confidence measure demonstrated improvement with regular performance of physical activity and consistent use of the wearable smart device.

\section{Discussion}

This case study of the utilization of a fall-sensing wearable that will automatically deploy airbags when needed to protect the hips demonstrates the impact a wearable safety device can have as part of a fall mitigation care plan for an at risk individual living in the home environment. Enabling older adults to live and age in place in their own home decreases healthcare costs and increases quality of life. Traditional methods of fall mitigation should continue to be implemented in environmental adaptations, remediation, or compensation for physical or cognitive deficits and mitigation strategies. As the older adult population grows, so will the number of those at risk of serious fall injuries including the devastating event of a hip fracture. The introduction of newer and adjunctive options to address gaps in fall risk mitigation provide an increased sense of security and peace of mind for clients as well as their caregivers and families. On-demand or automatic types of safety devices with human motion monitoring will offer increased safety with reduced incidence of ED visits, reduce response time to falls and increase peace-of-mind for users/families of those most at risk of falls and fractures. Awareness of the added safety component for an at-risk individual can offer inherent reduction of fear. Reduction in fear of falling and reduction of fear of fall injuries leads to increased engagement in physical activity and mobility. Cognition is an important consideration in the fear cycle for these types of individuals and was an influence on the outcomes of this case study.

\section{Conclusion and Implications}

Identification of fall risk factors and the underlying sources of risk for an individual older adult is imperative to addressing the devastating risk of fall injuries. The mitigation of fall and injury from fall risk is best achieved in interdisciplinary and multi-modal approaches customized for the person's identified areas of weakness or need. Traditional methods of fall risk mitigation can be effective at reducing the risk of falls and fall injuries, though in most cases the risk of falling remains. The known risk of falling and subsequent risk of fall injuries can embed a 
sense of fear into the individual as well as onto those that care for them. The utilization of new technologies should be considered in offering older adults at risk of falls and fractures the ability to move with decreased fear of fall injury. This case study highlights the effectiveness of person-centered risk identification, resource management and interdisciplinary care to reduce risk of falls and fall injuries for successful remediation and compensation of specific identified risk factors and offer the ability to age in place for continued maintenance of quality of life.

\section{References}

Adeyemi, A., \& Delhougne, G. (2020). Incidence and economic burden of intertrochanteric fracture: a medicare claims database analysis. JBJS Open Access, 4(1), e0045. https://doi.org/10.2106/JBJS.OA.18.00045

Centers for Disease Control and Prevention. (2020). CDC hip fractures among older adults. Retrieved September 24, 2020, from https://www.cdc.gov/homeandrecreationalsafety/falls/adulthipfx.html

Get Your Patients Off to a Healthy Start in 2020. (2020). Centers for Medicare and Medicaid services; annual wellness visit. Retrieved September 21, 2020, from https://www.cms.gov/medicarepreventionprevntiongeninfohealth-observance-messages/get-your-patients-he althy-start-2020

Important Facts About Falls. (2020). Centers for disease control and prevention; home and recreational safety. Retrieved September 21, 2020, from https://www.cdc.gov/homeandrecreationalsafety/falls/adultfalls.html

Jones, C., et al.. (1999). A 30-s chair-stand test as a measure of lower body strength in community-residing older adults. Research Quarterly for Exercise and Sport, 70(1), 113.

Kempen, G. I., et al.. (2008). The Short FES-I: a shortened version of the falls efficacy scale-international to assess fear of falling. Age Ageing, 37(1), 45-50.

Konstantinos, M., et al. (2018). Quality of life and psychological consequences in elderly patients after a hip fracture: a review. Clinical Interventions in Aging, 2018(13), 143-150.

Market Data Forecast. (2020). Global PERS Market Size, Share, Trends, Growth Analysis Report - Segmented By Type (Mobile Based, Landline Based, Standalone), End User (Home-Based Users, Senior Living Facilities, Assisted Living Facilities, Others), Region - Industry Forecast | 2020 to 2025.

Middleton, A., et al.. (2016). Self-selected and maximal walking speeds provide greater insight into fall status than walking speed reserve among community-dwelling older adults. Am J Phys Med Rehabil, 95(7), 475-482.

NHS. (2020). Are you at risk of falling?. NHS Health Body. Retrieved September 21, 2020, from https://www.nhs.uk/live-well/healthy-body/are-you-at-risk-of-falling/

Phelan, E., et al.. (2020). Assessment and Fall Risk in Primary Care Settings. Med Clin North Am, 99(2), 281-293.

Quigley, P., et al.. (2019). Innovation to protect hips from fall-related fracture. Phys Med Rehabil Res, 4, 1-4.

Shumway-Cook, A., et al.. (2000). Predicting the probability for falls in community-dwelling older adults using the Timed Up \& Go Test. Physical Therapy, 80(9), 896-903.

Thomas, J., \& Lane, J. V. (2005). A pilot study to explore the predictive validity of 4 measures of falls risk in frail elderly patients. Arch Phys Med Rehabil, 86, 1636-1640.

World Health Organization. (2007). WHO Global Report on Falls Prevention in Older Age. Geneva, Switzerland: World Health Organization A Global Report on Falls Prevention: Epidemiology of Falls. Retrieved September 21, 2020, https://www.who.int/ageing/projects/1.Epidemiology\%20of\%20falls\%20in\%20older\%20age.pdf

\section{Copyrights}

Copyright for this article is retained by the author(s), with first publication rights granted to the journal.

This is an open-access article distributed under the terms and conditions of the Creative Commons Attribution license (http://creativecommons.org/licenses/by/4.0/). 